Article

\title{
Effects of Prolonged Type 2 Diabetes on the Inner Retinal Layer and Macular Microvasculature: An Optical Coherence Tomography Angiography Study
}

\author{
Min-Woo Lee ${ }^{1}$, Woo-Hyuk Lee ${ }^{2} \mathbb{D}$, Cheon-Kuk Ryu ${ }^{2}$, Tae-Yeon Kim ${ }^{1}$, Hyung-Bin Lim ${ }^{2}$, \\ Young-Hoon Lee ${ }^{1}$ and Jung-Yeul Kim ${ }^{2,3, *}$ \\ 1 Department of Ophthalmology, Konyang University College of Medicine, Daejeon 35365, Korea; \\ bogus1105@gmail.com (M.-W.L.); skykty96@kyuh.ac.kr (T.-Y.K.); astrix001@gmail.com (Y.-H.L.) \\ 2 Department of Ophthalmology, Chungnam National University College of Medicine, Daejeon 35015, Korea; \\ lwhyuk@naver.com (W.-H.L.); chkryu6@gmail.com (C.-K.R.); cromfans@hanmail.net (H.-B.L.) \\ 3 Department of Ophthalmology, Chungnam National University Hospital, \#640 Daesa-dong, Jung-gu, \\ Daejeon 301-721, Korea \\ * Correspondence: kimjy@cnu.ac.kr; Tel.: +82-42-280-8433; Fax: +82-42-255-3745
}

Received: 26 May 2020; Accepted: 11 June 2020; Published: 13 June 2020

\begin{abstract}
Purpose: To identify the effects of prolonged type 2 diabetes (T2DM) on macular microcirculation and the inner retinal layer in diabetic eyes without clinical diabetic retinopathy (DR). Methods: 97, 92, and 57 eyes in the control, patients with T2DM $<10$ years (DM group one), and patients with $\mathrm{T} 2 \mathrm{DM} \geq 10$ years (DM group two) were enrolled. The ganglion cell-inner plexiform layer (GC-IPL) thickness and superficial vessel density (VD) were compared. Linear regression analyses were performed to identify factors associated with VD in T2DM patients. Results: GC-IPL thicknesses in the control, DM group one, and DM group two were $84.58 \pm 0.89,83.49 \pm 0.70$, and $79.04 \pm 0.96 \mu \mathrm{m}$, respectively $(p<0.001)$. The VDs of the full area were $20.32 \pm 0.15,19.46 \pm 0.17$ and $18.46 \pm 0.23 \mathrm{~mm}^{-1}(p<0.001)$. Post-hoc analyses revealed that the VDs of the full area was significantly different in the control vs. DM group one $(p=0.001)$, control vs. DM group two $(p<0.001)$, and DM group one vs. DM group two $(p=0.001)$. Multivariate linear regression analyses revealed that DM duration ( $p=0.037)$, visual acuity $(p=0.013)$, and GC-IPL thickness $(p<0.001)$ were significantly associated with the VD of T2DM patients. Conclusions: We confirmed GC-IPL thinning and decreased superficial VD in the macular areas using OCTA in T2DM patients. Patients with $\mathrm{T} 2 \mathrm{DM} \geq 10$ years exhibited significantly more severe macular microcirculation impairment compared to patients with $\mathrm{T} 2 \mathrm{DM}<10$ years and normal controls.
\end{abstract}

Keywords: diabetes; optical coherence tomography angiography; ganglion cell-inner plexiform layer; vessel density

\section{Introduction}

Type 2 diabetes (T2DM) is a major global health problem that is increasing in prevalence due to lifestyle changes [1-4]. The International Diabetes Federation estimated that there were 451 million people with diabetes worldwide in 2017, and expected to increase to 693 million by 2045 [4]. This increase in T2DM could cause an increase in diabetic retinopathy (DR) [5]. DR, which is the leading cause of blindness in the working-age population, is known to be associated with ischemic damage on retina following to change in the microvasculature [6-8]. Such retinal damage is also found in patients without clinical DR. Previous studies have found that retinal damage caused by T2DM could result in thinning of the ganglion cell-inner plexiform layer (GC-IPL) and peripapillary retinal nerve fiber layer (pRNFL) in T2DM patients without clinical DR [9-11]. Such inner retinal thinning reflects diabetic 
retinal neurodegeneration, an early event in DR pathogenesis activated by pathologic pathways such as polyols, hexosamine, or oxidative stress [12,13]. Recently, with the development of devices that are able to observe retinal microvasculature and retinal perfusion in detail, various studies on retinal microvasculature damage in patients with T2DM have been reported.

Optical coherence tomography angiography (OCTA) is a noninvasive imaging technique used to examine the microvasculature of the retina and choroid, which enables visualization of the fine vasculatures of multiple layers. Several studies using OCTA have reported reduced macular microcirculation in patients with T2DM [14-17]. Cao et al. [14] found that the parafoveal vessel density (VD) of both the superficial and deep capillary plexuses was decreased in diabetic eyes without clinical DR. Li et al. [15] reported foveal avascular zone (FAZ) enlargement and an increased FAZ perimeter in T2DM patients without clinical DR compared to normal controls. As such, retina can be damaged by T2DM in various forms and cannot be concluded to be a normal state even if clinical DR is not observed. Therefore, the state of the retina without clinical DR might be different depending on various factors, but few studies have explored how T2DM duration affects retinal microvasculature.

The purpose of this study was to identify the effects of prolonged T2DM on macular microvasculature and the inner retinal layer by comparing the superficial VD and GC-IPL thickness of patients with $\mathrm{T} 2 \mathrm{DM}<10$ years and patients with $\mathrm{T} 2 \mathrm{DM} \geq 10$ years.

\section{Methods}

\subsection{Patients}

This observational, cross-sectional study adhered to the tenets of the Declaration of Helsinki and was approved by the Institutional Review Board of Chungnam National University Hospital, Daejeon, Republic of Korea. We reviewed the charts of patients with T2DM who visited the retina clinic of Chungnam National University Hospital for DR checkups from March 2017 to December 2019. We recorded detailed histories and best-corrected visual acuity (BCVA), intraocular pressure (IOP), spherical equivalent (SE), and axial length (using an IOLMaster; Carl Zeiss, Jena, Germany, version 5.02). Subjects were divided into three groups: Control, patients with T2DM $<10$ years (DM group one), and patients with $\mathrm{T} 2 \mathrm{DM} \geq 10$ years (DM group two). Exclusion criteria were a history of systemic disease other than T2DM; any ophthalmic disease such as glaucoma, retinal diseases, or neuro-ophthalmic diseases; axial length $\geq 26.0 \mathrm{~mm}$; any prior intraocular surgery except cataract extraction; a BCVA < 0.7; and an IOP $>21 \mathrm{mmHg}$. We also excluded patients with clinical evidence of DR such as retinal hemorrhage or microaneurysms. One eye was randomly selected in patients satisfied with inclusion criteria.

\subsection{OCT Measurements}

We performed SD-OCT (Cirrus HD OCT 5000, version 10.0; Carl Zeiss Meditec, Dublin, CA, USA) using 512 by 128 macular cube and 200 by 200 optic disc cube scanning protocols to measure GC-IPL and pRNFL thicknesses. The ganglion cell analysis algorithm automatically measured GC-IPL thickness by identifying the outer boundaries of the RNFL and the IPL of the macula using the three-dimensional information from the macular cube scan. The average, minimum, and six sectoral (superior, superonasal, inferonasal, inferior, inferotemporal, and superotemporal) GC-IPL thicknesses were analyzed. Images with a signal strength $<7$, obvious decentration, or segmentation errors were excluded.

\subsection{VD Measurement Using OCTA}

OCTA images were obtained by an experienced examiner using a Cirrus HD-OCT 5000 along with AngioPlex software (Carl Zeiss Meditec). AngioPlex yields high-resolution retinal microvascular images using a center wavelength of $840 \mathrm{~nm}$, taking 68,000 A-scans per second. The instrument provides sensitivity and accuracy by incorporating the optical microangiography (OMAG) algorithm 
and retinal tracking technology. We obtained foveal centered scan area of 3 by $3 \mathrm{~mm}$ pattern, and all scans were analyzed using en face OCTA images generated automatically by the OMAG algorithm used in AngioPlex software. The VD of the superficial capillary plexus, which spanned from the internal limiting membrane to the IPL, was measured automatically by the software. The software quantified VD via the Early Treatment of Diabetic Retinopathy Study subfields (Figure 1).

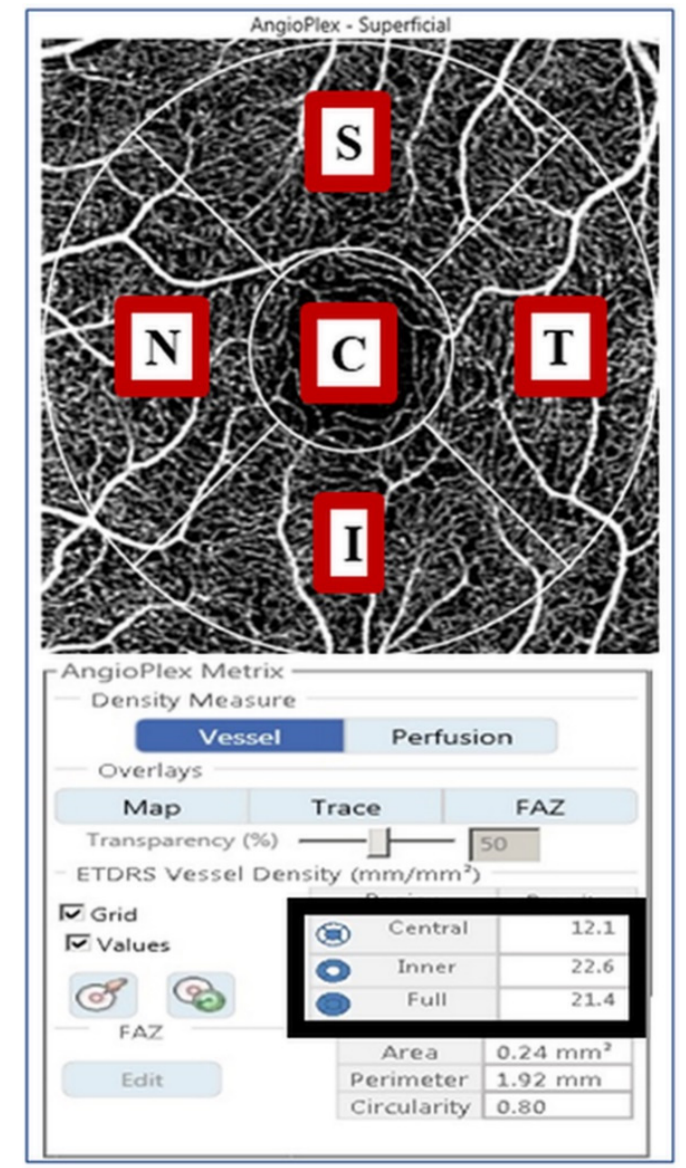

Figure 1. A 3 by $3 \mathrm{~mm}$ optical coherence tomography angiography image centered on the fovea. The en face image of the superficial layer is overlaid with the Early Treatment of Diabetic Retinopathy Study grid. The measurement tool (AngioPlex software, V. 10.0; Carl Zeiss Meditec) yields vessel density measurements in individual subfields. The black box contains quantitative vessel density measurements of the central, inner, and full areas. $C$, center; $S$, superior; T, temporal; I, inferior; N, nasal.

All images were checked and verified by two blinded observers (M.W.L. and W.H.L.), and any images exhibiting fixation loss, segmentation errors, motion artifacts, or signal strengths $<8$ were excluded.

\subsection{Statistical Analysis}

Demographic characteristics and ocular parameters were compared via one-way analysis of variance with the post-hoc Bonferroni correction and the chi-squared test. Univariate and multivariate linear regression analyses were performed to identify factors associated with superficial macular VD in patients with T2DM. All statistical analyses were performed with the aid of SPSS software (version 18.0; IBM Corp., Armonk, NY, USA). 


\section{Results}

\subsection{Demographics}

A total of 246 eyes were enrolled: 97 in the control group, 92 in DM group one, and 57 in DM group two. The mean ages of each group were $63.82 \pm 0.44,62.43 \pm 1.09$, and $64.12 \pm 1.11$ years, respectively, which did not show a significant difference $(p=0.356)$ (Table 1$)$.

Table 1. Demographics and clinical characteristics.

\begin{tabular}{ccccc}
\hline & $\begin{array}{c}\text { Normal Controls } \\
(\boldsymbol{n}=\mathbf{9 7})\end{array}$ & $\begin{array}{c}\text { DM Group 1 } \\
(\boldsymbol{n}=\mathbf{9 2})\end{array}$ & $\begin{array}{c}\text { DM Group 2 } \\
(\boldsymbol{n}=\mathbf{5 7})\end{array}$ & $\boldsymbol{p}$-Value \\
\hline Age (mean \pm SE, years) & $63.82 \pm 0.44$ & $62.43 \pm 1.09$ & $64.12 \pm 1.11$ & 0.356 \\
Sex (male, \%) & $44(45.4 \%)$ & $35(38.0 \%)$ & $28(49.1 \%)$ & 0.371 \\
Laterality (right, $\%)$ & $55(56.7 \%)$ & $46(50.0 \%)$ & $28(49.1 \%)$ & 0.555 \\
BCVA (mean \pm SE, logMAR) & $-0.019 \pm 0.006$ & $0.002 \pm 0.007$ & $0.003 \pm 0.007$ & $0.07 \pm 0.18$ \\
SE (mean \pm SE, diopters) & $0.06 \pm 0.11$ & $-0.36 \pm 0.14$ & $15.47 \pm 0.36$ & 0.060 \\
IOP (mean \pm SE, mmHg) & $15.64 \pm 0.27$ & $15.96 \pm 0.32$ & $23.51 \pm 0.11$ & 0.196 \\
Axial length (mean \pm SE, mm) & $23.57 \pm 0.07$ & $23.72 \pm 0.08$ & $14.61 \pm 0.57$ & $<0.001$ \\
DM duration (mean \pm SE, years) & 0 & $3.51 \pm 0.29$ & $7.04 \pm 0.12$ & 0.397 \\
HbA1C (mean \pm SE, \%) & N/A & $6.90 \pm 0.10$ & $248.49 \pm 2.44$ & 0.317 \\
CMT (mean \pm SE, $\mu$ m) & $250.22 \pm 1.99$ & $246.04 \pm 1.93$ & $92.49 \pm 1.34$ \\
pRNFL thickness (mean \pm SE, $\mu$ m) & $96.71 \pm 0.88$ & $94.82 \pm 1.07$ & 0.032 \\
\hline
\end{tabular}

$\mathrm{SE}=$ standard errors; BCVA = best-corrected visual acuity; $\mathrm{SE}=$ spherical equivalent; $\mathrm{IOP}=$ intraocular pressure; DM = diabetes; $\mathrm{CMT}=$ central macular thickness; and pRNFL = peripapillary retinal nerve fiber layer. DM group 1 = patients with type 2 diabetes $<10$ years, DM group $2=$ patients with type 2 diabetes $\geq 10$ years. Values in boldface $(p<0.05)$ are statistically significant.

Sex, spherical equivalent, IOP, and axial length were not also significantly different among the groups. Visual acuity showed a significant difference among the groups $(p=0.027)$, but this difference disappeared on post-hoc analyses (control vs. DM group one, $p=0.056$; control vs. DM group two, $p=0.088$; DM group one vs. DM group two, $p=1.000$ ). The T2DM durations were $3.51 \pm 0.29$ and $14.61 \pm 0.57$ years $(p<0.001)$, and the HbA1c were $6.90 \pm 0.10$ and $7.04 \pm 0.12 \%(p=0.397)$ in DM group one and DM group two, respectively.

\subsection{GC-IPL Thickness in Each Group}

Average GC-IPL thicknesses in the control group, DM group one, and DM group two were $84.58 \pm 0.89,83.49 \pm 0.70$, and $79.04 \pm 0.96 \mu \mathrm{m}$, respectively, which showed a significant difference $(p<0.001)$ (Table 2).

Table 2. Ganglion cell-inner plexiform layer thickness in each group.

\begin{tabular}{ccccc}
\hline & Control & DM Group 1 & DM Group 2 & $p$-Value \\
\hline Average & $84.58 \pm 0.89$ & $83.49 \pm 0.70$ & $79.04 \pm 0.96$ & $<0.001$ \\
Minimum & $79.61 \pm 0.91$ & $80.03 \pm 0.94$ & $73.39 \pm 1.70$ & $<0.001$ \\
Sector & & & & \\
Superior & $83.89 \pm 0.82$ & $84.39 \pm 0.71$ & $78.98 \pm 1.11$ & $<0.001$ \\
Superotemporal & $82.13 \pm 1.03$ & $81.91 \pm 0.78$ & $78.81 \pm 1.03$ & 0.014 \\
Inferotemporal & $82.89 \pm 0.75$ & $83.05 \pm 0.78$ & $79.54 \pm 0.99$ & 0.008 \\
Inferior & $80.88 \pm 0.76$ & $81.13 \pm 0.73$ & $77.26 \pm 0.88$ & 0.001 \\
Inferonasal & $83.27 \pm 0.79$ & $83.61 \pm 0.79$ & $79.07 \pm 1.08$ & 0.001 \\
Superonasal & $85.73 \pm 0.82$ & $86.12 \pm 0.82$ & $80.46 \pm 1.45$ & $<0.001$ \\
\hline
\end{tabular}

DM group 1 = patients with type 2 diabetes $<10$ years, DM group $2=$ patients with type 2 diabetes $\geq 10$ years. Values in boldface $(p<0.05)$ are statistically significant. All values are expressed as the mean \pm standard errors $(\mu \mathrm{m})$.

On post-hoc analyses, significant differences were observed in the control vs. DM group two, and DM group one vs. DM group two ( $p<0.001$ and $p=0.002$, respectively). Additionally, the GC-IPL 
thicknesses of all six sectors differed significantly among the groups. On post-hoc analyses, control vs. DM group two and DM group one vs. DM group two were significantly different as same as the result of comparison in the average GC-IPL thickness.

\subsection{Superficial Macular VD in Each Group}

The average signal strengths of OCTA images were $9.71 \pm 0.05,9.67 \pm 0.05$, and $9.61 \pm 0.08$ in the control, DM group one, and DM group two, respectively $(p=0.165)$. The VDs of the full areas were $20.32 \pm 0.15,19.46 \pm 0.17$, and $18.46 \pm 0.23 \mathrm{~mm}^{-1}$ in the control, DM group one, and DM group two, respectively, which showed a significant difference $(p<0.001)$ (Table 3) (Figure 2).

Table 3. Superficial macular vessel density in each group using optical coherence tomography angiography.

\begin{tabular}{ccccc}
\hline & Control & DM Group 1 & DM Group 2 & $p$-Value \\
\hline Full area & $20.32 \pm 0.15$ & $19.46 \pm 0.17$ & $18.46 \pm 0.23$ & $<0.001$ \\
Inner area & $21.70 \pm 0.14$ & $20.77 \pm 0.17$ & $19.78 \pm 0.24$ & $<0.001$ \\
Central area & $9.41 \pm 0.28$ & $9.34 \pm 0.30$ & $8.27 \pm 0.31$ & 0.027 \\
Sector & & & & \\
Superior & $21.58 \pm 0.19$ & $20.65 \pm 0.22$ & $19.72 \pm 0.30$ & $<0.001$ \\
Temporal & $21.62 \pm 0.14$ & $20.76 \pm 0.19$ & $20.05 \pm 0.23$ & $<0.001$ \\
Inferior & $21.74 \pm 0.15$ & $20.75 \pm 0.22$ & $19.89 \pm 0.30$ & $<0.001$ \\
Nasal & $21.83 \pm 0.22$ & $20.91 \pm 0.21$ & $19.49 \pm 0.39$ & $<0.001$
\end{tabular}

DM group 1 = patients with type 2 diabetes $<10$ years, DM group 2 = patients with type 2 diabetes $\geq 10$ years. Values in boldface $(p<0.05)$ are statistically significant. All values are expressed as the mean \pm standard errors $\left(\mathrm{mm}^{-1}\right)$.

(a)

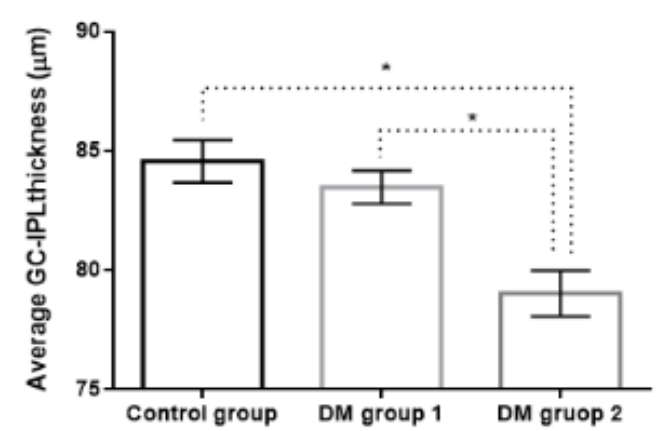

(b)

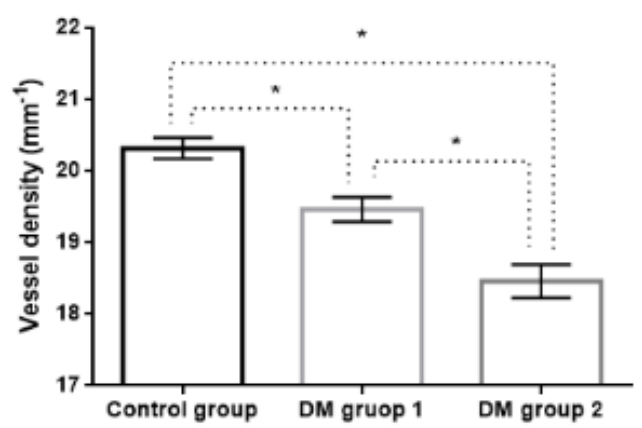

Figure 2. Bar graph with standard errors of average ganglion cell-inner plexiform layer (GC-IPL) thickness (a) and vessel density of $3 \mathrm{~mm}$ full area (b) in each group. * Statistically significant difference. DM group 1, patients with type 2 diabetes $<10$ years; DM group 2, patients with type 2 diabetes $\geq 10$ years.

The VDs of the inner, and central areas and four sectors also differed significantly. On post-hoc analyses, the VDs of the full, and inner areas, and four sectors were significantly different in the control vs. DM group one, control vs. DM group two, and DM group one vs. DM group two.

\subsection{Factors Associated with Superficial Macular VD in T2DM Patients}

On univariate linear regression analyses, the duration of T2DM $(B=-0.07, p=0.002)$, visual acuity $(\mathrm{B}=-5.13, p=0.028)$, and the average GC-IPL thickness $(\mathrm{B}=0.08, p<0.001)$ were significant factors associated with the full VD of macular area (Table 4, Figure 3). 
Table 4. Univariate and multivariate linear regression analyses determining factors associated with superficial macular vessel density in patients with type 2 diabetes.

\begin{tabular}{ccccc}
\hline \multicolumn{2}{c}{ Univariate } & \multicolumn{2}{c}{ Multivariate } \\
\hline B (95\% CI) & $p$-Values & B (95\% CI) & $p$-Values \\
\hline Age & $-0.01(-0.03-0.03)$ & 0.997 & & \\
Sex & $-0.33(-0.77-0.11)$ & 0.138 & & \\
DM duration & $-0.07(-0.11-0.03)$ & 0.002 & $-0.05(-0.09-0.01)$ & 0.037 \\
BCVA & $-5.13(-9.69--0.57)$ & 0.028 & $-5.39(-9.62--1.15)$ & 0.013 \\
SE & $0.02(-0.19-0.23)$ & 0.825 & & \\
IOP & $0.04(-0.06-0.14)$ & 0.442 & & \\
Axial length & $-0.03(-0.39-0.33)$ & 0.866 & & \\
HbA1C & $-0.27(-0.56-0.02)$ & 0.069 & $-0.06(-0.35-0.23)$ & \\
CMT & $0.01(-0.02-0.02)$ & 0.989 & & 0.226 \\
pRNFL & $0.02(-0.01-0.05)$ & 0.096 & $-0.02(-0.05-0.01)$ & $<0.001$ \\
GC-IPL & $0.08(0.05-0.12)$ & $<0.001$ & $0.07(0.04-0.11)$ & \\
\hline
\end{tabular}

$\mathrm{BCVA}=$ best-corrected visual acuity; $\mathrm{SE}=$ spherical equivalent; IOP $=$ intraocular pressure; $\mathrm{DM}=$ diabetes; $\mathrm{CMT}$ = central macular thickness; $\mathrm{pRNFL}=$ peripapillary retinal nerve fiber layer; and GC-IPL = ganglion cell-inner plexiform layer. Values in boldface $(p<0.05)$ are statistically significant.

(a)

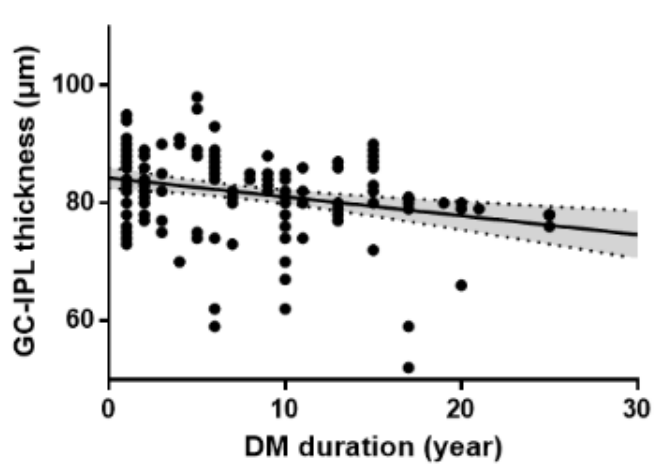

(b)

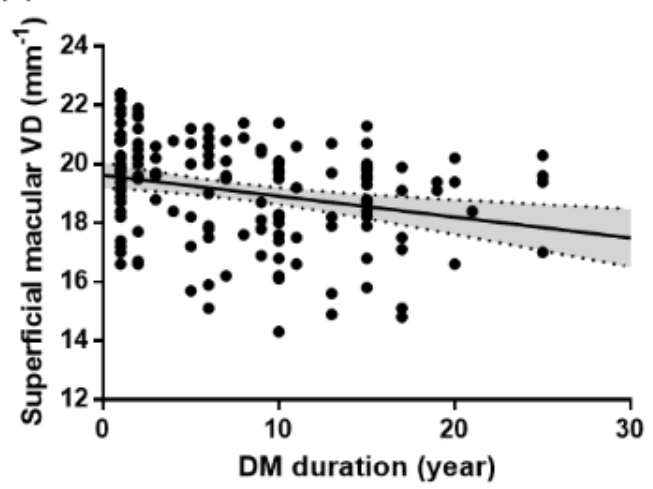

Figure 3. Scatterplots and linear regression analyses between the duration of type 2 diabetes (DM) and GC-IPL thickness $(r=0.284, p<0.001)$ (a) and superficial macular vessel density (VD) $(r=0.259$, $p=0.001)(\mathbf{b})$ in patients with DM.

Multivariate analyses revealed that T2DM duration $(B=-0.05, p=0.037)$, visual acuity $(B=-5.39$, $p=0.013)$, and average GC-IPL thickness $(B=0.07, p<0.001)$ were significant factors, in agreement with the univariate analyses (Figure 4).

(a)

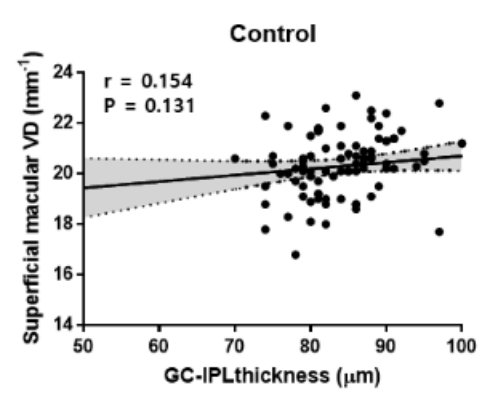

(b)

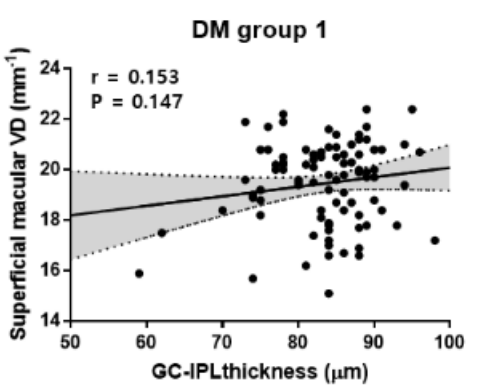

(c)

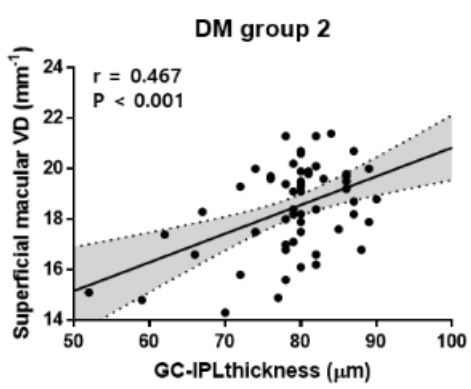

Figure 4. Scatterplots and linear regression analyses between superficial macular VD and GC-IPL thickness for each group. (a) control group, (b) DM group 1, (c) DM group 2. DM group 1 = patients with type 2 diabetes $<10$ years, DM group 2 = patients with type 2 diabetes $\geq 10$ years. 


\section{Discussion}

Previous studies have reported reduced retinal blood circulation times and oxygen maldistributions in the major peripapillary arteries and veins of T2DM patients without DR. [18,19] Others have found that some diabetic eyes without any visible microvascular findings on ophthalmoscopy exhibited microaneurysms on OCTA images [14,20]. Additionally, diabetic retinal neurodegeneration can cause inner retinal thinning and delayed implicit time of multifocal electroretinography prior to DR development $[12,13,21]$. As such, retinal and circulatory damage caused by T2DM may present in various forms and to various extents even in eyes without clinical DR. We focused on the GC-IPL and microvasculature of the macular area, the most important area on vision, and evaluated the effects of prolonged T2DM on macular microvasculature in eyes without clinical DR. We observed thinning of GC-IPL and reduced superficial VD of the macular area in patients with T2DM, and the patients with prolonged DM had more prominent damage. Additionally, DM duration, visual acuity, and GC-IPL thickness were significantly associated with superficial macular VD in T2DM patients.

Previous studies have reported GC-IPL damage in patients with T2DM [22-24]. Ng et al. [22] reported that subjects with T2DM but without DR had an average GC-IPL thinning of $4.37 \mu \mathrm{m}$, compared to controls. Lim et al. [23] found that the estimated reduction rate of the average GC-IPL thickness in T2DM patients without DR $(-0.627 \mu \mathrm{m} /$ year $)$ was 2.26 -fold faster than that in normal individuals $(-0.277 \mu \mathrm{m} /$ year $)$. We obtained similar results: The GC-IPL was significantly thinner in T2DM patients than controls. In patients with T2DM, diabetic retinal neurodegeneration, which may develop before any definite microvascular change as mentioned above, manifests as neuronal apoptosis and reactive gliosis. Retinal ganglion and amacrine cells are known to be the first neurons to exhibit diabetes-induced apoptosis, which could result in thinning of the inner retinal layers including the GC-IPL before the prominent retinal vascular changes of DR are apparent $[11,13,25]$. Such thinning could indirectly provide information on central nervous system (CNS) damage caused by T2DM, which is an easily accessible and non-invasive way. The retina is ontogenically brain-derived tissue, and diabetic retinal neurodegeneration and neurodegenerative diseases such as Alzheimer's disease are known to share several pathogenic pathways, such as insulin signaling impairment, the accumulation of advanced glycation end-products, and an increase in oxidative stress [26]. Therefore, even without DR, GC-IPL monitoring, which could provide information on CNS damage as well as retina, is crucial especially to patients with prolonged T2DM patients.

We observed a decreased superficial macular VD in T2DM patients. Zeng et al. [16] identified significantly decreased parafoveal and perifoveal VDs of both the superficial and deep capillary plexus in T2DM patients without DR, compared to controls. Alibhai et al. [17] reported a significantly larger FAZ area and lower binarized flow index of both the superficial and deep retinal layers in diabetic eyes without DR, compared to normal controls. In the retina, neuronal, glial, and vascular cells are closely connected in the neurovascular unit, and an autoregulatory response of the neurovascular unit to complex circulatory and neural cues is essential to regulate blood flow through the inner retina because of lacking autonomic innervation of the intra-retinal vasculature $[27,28]$. Therefore, the decreased VD may be affected by the disruption of neurovascular autoregulation which could get damaged by diabetic retinal neurodegeneration, but further studies to prove the direct relationship between autoregulation and macular VD are needed to confirm this [13,29]. Additionally, endothelial cell injuries by chronic hyperglycemia can cause acellular retinal capillaries, which may result in reduced macular VD $[13,16]$. Thus, T2DM patients, even those lacking clinical DR, would have impaired macular microvasculature.

The superficial macular VDs differed significantly between DM group one and DM group two, and DM duration was significantly associated with the superficial macular VD of T2DM patients. Whereas, HbA1C was not significantly related to superficial macular VD. Sohn et al. [30] reported that the progressive neuroretinal degeneration was primarily related to DM duration and not to $\mathrm{HbA} 1 \mathrm{C}$. Diabetic retinal neurodegeneration is closely related to macular microvasculature by the neurovascular unit, which may result in the appearance of a similar trend. Although clinical information on the 
relationship between glycemic control and retinal neurodegeneration and macular microvasculature is not available, the major components of the renin-angiotensin system have been identified to be overexpressed in the retina of DM patients, and the blockade of the system in experimental models of DM attenuated retinal neurodegeneration [31,32]. Thus, even under the relatively good glycemic control, prolonged DM may exacerbate macular microvasculature impairment. So, physicians should not conclude that the retina of DM patients is under a stable and safe state even they are not showing DR and under good glycemic control.

Samara et al. [33] found a positive correlation of VD with visual acuity at the level of both the superficial and deep vascular networks in patients with DR. Li et al. [15] reported that logMAR BCVA and FAZ showed a significant negative correlation with superficial VD in DM patients without DR, suggesting that the FAZ and superficial VD are potentially sensitive factors that affect the visual acuity in DM subjects. Our study also found that visual acuity was significantly associated with superficial macular VD in T2DM patients. In the situation of impairment of choroidal circulation due to failure of the autoregulatory mechanism in DM, the metabolic demands of the outer retina including photoreceptor would become more dependent on the vascular supply from the inner retina, which might directly link superficial macular VD to visual acuity [34]. Impairment of vision-related quality of life in T2DM patients such as decreased hue discrimination, decreased contrast sensitivity, delayed dark adaptation, and reduced visual field sensitivity, which was identified to the consequences of diabetic retinal neurodegeneration, would be also related to macular microvasculature impairment, although further studies are needed to confirm this [21,35-37]. Besides a promising tool for monitoring and follow-up of macular ischemia, superficial macular VD could be one of the significant factors reflecting the visual function in patients with DM.

Vujosevic et al. [38] found a significant correlation between OCTA parameters such as perfusion density or VD and RNFL thickness in the peripapillary area. Mase et al. [39] explained this correlation that radial peripapillary plexus is the most important structure in maintaining RNFL integrity. We also found that the average GC-IPL thickness correlated significantly with the superficial macular VD, but only in DM group two. Notably, Lim et al. [40] reported that the average GC-IPL thickness was significantly correlated with the superficial macular VD only in subjects with hypertension $\geq 10$ years. The ganglion cell bodies in the macular area are multilayered, and are 10- to 20-fold thicker than their axons [41]. Additionally, whereas the peripapillary area consists of capillaries with long straight paths and rare anastomotic connections, the superficial capillary plexus of the macula has a dense capillary network with numerous anastomoses, which may compensate to some extent for hypoxic damage caused by systemic disease [42-44]. Therefore, enough time for damages by diabetic retinal neurodegeneration of ganglion cell bodies in the macular area might be needed before a direct relationship between GC-IPL and superficial macular VD is evident [45].

Our study had several limitations. First, the retrospective nature of the work inevitably introduces some selection bias. Second, we did not analyze the VD of the deep capillary plexus because the automatic AngioPlex microcirculation parameters pertain only to the superficial capillary plexus. However, analysis of the superficial capillary plexus is more accurate than that of the deep capillary plexus because of the projection artifacts almost occurring in current technology [46]. Third, as subjects with $\mathrm{T} 2 \mathrm{DM} \geq 10$ years had a higher probability of having DR or other systemic diseases than other groups, there were fewer cases in DM group two meeting the inclusion criteria than other groups. The strength of our study was that we enrolled OCTA images with $S S \geq 8$, allowing accurate analyses. Likewise, this is the first study to identify the impact of prolonged DM on the macular microvasculature besides the inner retinal layer by dividing groups according to the DM duration.

\section{Conclusions}

In conclusion, we confirmed GC-IPL thinning and decreased superficial VD on the macular areas using OCTA in T2DM patients without clinical DR. Patients with T2DM $\geq 10$ years exhibited significantly more severe macular microvasculature impairment than T2DM patients with relatively short disease 
durations or normal controls, even they were under relatively good glycemic control. Even in the absence of clinical DR, physicians should consider constant diabetic retinal neurodegeneration and macular microvasculature impairment in T2DM patients, and monitor GC-IPL and macular VD using OCTA.

Author Contributions: Design and conduct of the study M.-W.L., J.-Y.K.; Collection of data M.-W.L., W.-H.L., C.-K.R., J.-Y.K.; Analysis and interpretation of data M.-W.L., W.-H.L., T.-Y.K., H.-B.L., J.-Y.K.; Writing the article M.-W.L., J.-Y.K.; Critical revision of the article M.-W.L., W.-H.L., Y.-H.L., J.-Y.K.; Final approval of the article M.-W.L., W.-H.L., C.-K.R., T.-Y.K., H.-B.L., Y.-H.L., J.-Y.K. All authors have read and agreed to the published version of the manuscript.

Funding: This research received no external funding.

Conflicts of Interest: The authors declare no conflict of interest.

\section{References}

1. Shaw, J.E.; Sicree, R.A.; Zimmet, P.Z. Global estimates of the prevalence of diabetes for 2010 and 2030. Diabetes Res. Clin. Pract. 2010, 87, 4-14. [CrossRef] [PubMed]

2. Danaei, G.; Lu, Y.; Singh, G.M.; Carnahan, E.; Stevens, G.A.; Cowan, M.J.; Farzadfar, F.; Lin, J.K.; Finucane, M.M.; Rao, M.; et al. Cardiovascular disease, chronic kidney disease, and diabetes mortality burden of cardiometabolic risk factors from 1980 to 2010: A comparative risk assessment. Lancet Diabetes Endocrinol. 2014, 2, 634-647.

3. Yang, W.; Lu, J.; Weng, J.; Jia, W.; Ji, L.; Xiao, J.; Shan, Z.; Liu, J.; Tian, H.; Ji, Q.; et al. Prevalence of diabetes among men and women in China. N. Engl. J. Med. 2010, 362, 1090-1101. [CrossRef] [PubMed]

4. Cho, N.; Shaw, J.; Karuranga, S.; Huang, Y.; da Rocha Fernandes, J.D.; Ohlrogge, A.W.; Malanda, B. IDF Diabetes Atlas: Global estimates of diabetes prevalence for 2017 and projections for 2045. Diabetes Res. Clin. Pract. 2018, 138, 271-281. [CrossRef] [PubMed]

5. Yau, J.W.; Rogers, S.L.; Kawasaki, R.; Lamoureux, E.L.; Kowalski, J.W.; Bek, T.; Chen, S.J.; Dekker, J.M.; Fletcher, A.; Grauslund, J.; et al. Global prevalence and major risk factors of diabetic retinopathy. Diabetes Care 2012, 35, 556-564. [CrossRef] [PubMed]

6. Klein, R.; Klein, B.E.; Moss, S.E.; Davis, M.D.; DeMets, D.L. The Wisconsin Epidemiologic Study of Diabetic Retinopathy: III. Prevalence and risk of diabetic retinopathy when age at diagnosis is 30 or more years. Arch. Ophthalmol. 1984, 102, 527-532. [CrossRef]

7. Durham, J.T.; Herman, I.M. Microvascular modifications in diabetic retinopathy. Curr. Diabetes Rep. 2011, 11, 253-264. [CrossRef]

8. Barot, M.; Gokulgandhi, M.R.; Patel, S.; Mitra, A.K. Microvascular complications and diabetic retinopathy: Recent advances and future implications. Future Med. Chem. 2013, 5, 301-314. [CrossRef]

9. Vujosevic, S.; Midena, E. Retinal layers changes in human preclinical and early clinical diabetic retinopathy support early retinal neuronal and Müller cells alterations. J. Diabetes Res. 2013, 2013. [CrossRef]

10. Carpineto, P.; Toto, L.; Aloia, R.; Ciciarelli, V.; Borrelli, E.; Vitacolonna, E.; di Nicola, M.; di Antonio, L.; Mastropasqua, R. Neuroretinal alterations in the early stages of diabetic retinopathy in patients with type 2 diabetes mellitus. Eye 2016, 30, 673. [CrossRef]

11. Chhablani, J.; Sharma, A.; Goud, A.; Peguda, H.K.; Rao, H.L.; Begum, V.U.; Barteselli, G. Neurodegeneration in type 2 diabetes: Evidence from spectral-domain optical coherence tomography. Investig. Ophthalmol. Vis. Sci. 2015, 56, 6333-6338. [CrossRef] [PubMed]

12. Simó, R.; Hernández, C. Neurodegeneration in the diabetic eye: New insights and therapeutic perspectives. Trends Endocrinol. Metab. 2014, 25, 23-33. [CrossRef] [PubMed]

13. Simó, R.; Stitt, A.W.; Gardner, T.W. Neurodegeneration in diabetic retinopathy: Does it really matter? Diabetologia 2018, 61, 1902-1912. [CrossRef] [PubMed]

14. Cao, D.; Yang, D.; Huang, Z.; Zeng, Y.; Wang, J.; Hu, Y.; Zhang, L. Optical coherence tomography angiography discerns preclinical diabetic retinopathy in eyes of patients with type 2 diabetes without clinical diabetic retinopathy. Acta Diabetol. 2018, 55, 469-477. [CrossRef] [PubMed]

15. Li, Z.; Alzogool, M.; Xiao, J.; Zhang, S.; Zeng, P.; Lan, Y. Optical coherence tomography angiography findings of neurovascular changes in type 2 diabetes mellitus patients without clinical diabetic retinopathy. Acta Diabetol. 2018, 55, 1075-1082. [CrossRef] [PubMed] 
16. Zeng, Y.; Cao, D.; Yu, H.; Yang, D.; Zhuang, X.; Hu, Y.; Li, J.; Yang, J.; Wu, Q.; Liu, B.; et al. Early retinal neurovascular impairment in patients with diabetes without clinically detectable retinopathy. Br. J. Ophthalmol. 2019, 103, 1747-1752. [PubMed]

17. Alibhai, A.Y.; Moult, E.M.; Shahzad, R.; Rebhun, C.B.; Moreira-Neto, C.; McGowan, M.; Lee, D.; Lee, B.; Baumal, C.R.; Witkin, A.J.; et al. Quantifying microvascular changes using OCT angiography in diabetic eyes without clinical evidence of retinopathy. Ophthalmol. Retin. 2018, 2, 418-427. [CrossRef]

18. Arend, O.; Wolf, S.; Jung, F.; Bertram, B.; Pöstgens, H.; Toonen, H.; Reim, M. Retinal microcirculation in patients with diabetes mellitus: Dynamic and morphological analysis of perifoveal capillary network. Br. J. Ophthalmol. 1991, 75, 514-518. [CrossRef]

19. Blair, N.P.; Wanek, J.; Felder, A.E.; Joslin, C.E.; Kresovich, J.K.; Lim, J.I.; Chau, F.Y.; Leiderman, Y.; Shahidi, M. Retinal oximetry and vessel diameter measurements with a commercially available scanning laser ophthalmoscope in diabetic retinopathy. Investig. Ophthalmol. Vis. Sci. 2017, 58, 5556-5563. [CrossRef]

20. Talisa, E.; Chin, A.T.; Bonini Filho, M.A.; Adhi, M.; Branchini, L.; Salz, D.A.; Baumal, C.R.; Crawford, C.; Reichel, E.; Witkin, A.J.; et al. Detection of microvascular changes in eyes of patients with diabetes but not clinical diabetic retinopathy using optical coherence tomography angiography. Retina 2015, 35, 2364-2370.

21. Bearse , M.A., Jr.; Adams, A.J.; Han, Y.; Schneck, M.E.; Ng, J.; Bronson-Castain, K.; Barez, S. A multifocal electroretinogram model predicting the development of diabetic retinopathy. Prog. Retin. Eye Res. 2006, 25, 425-448. [CrossRef] [PubMed]

22. Ng, D.S.; Chiang, P.P.; Tan, G.; Cheung, C.G.; Cheng, C.Y.; Cheung, C.Y.; Wong, T.Y.; Lamoureux, E.L. Mohammad K Ikram. Retinal ganglion cell neuronal damage in diabetes and diabetic retinopathy. Clin. Exp. Ophthalmol. 2016, 44, 243-250. [CrossRef] [PubMed]

23. Lim, H.B.; Shin, Y.I.; Lee, M.W.; Kim, J.Y. Ganglion Cell-Inner Plexiform Layer Damage in Diabetic Patients: 3-Year Prospective, Longitudinal, Observational Study. Sci. Rep. 2020, 10, 1-9. [CrossRef] [PubMed]

24. Kern, T.S.; Barber, A.J. Retinal ganglion cells in diabetes. J. Physiol. 2008, 586, 4401-4408. [CrossRef]

25. Carrasco, E.; Hernández, C.; Miralles, A.; Huguet, P.; Farrés, J.; Simó, R. Lower somatostatin expression is an early event in diabetic retinopathy and is associated with retinal neurodegeneration. Diabetes Care 2007, 30, 2902-2908. [CrossRef]

26. Simó, R.; Ciudin, A.; Simó-Servat, O.; Hernández, C. Cognitive impairment and dementia: A new emerging complication of type 2 diabetes-The diabetologist's perspective. Acta Diabetol. 2017, 54, 417-424. [CrossRef]

27. Gardner, T.W.; Davila, J.R. The neurovascular unit and the pathophysiologic basis of diabetic retinopathy. Graefe's Arch. Clin. Exp. Ophthalmol. 2017, 255, 1-6. [CrossRef]

28. Metea, M.R.; Newman, E.A. Signalling within the neurovascular unit in the mammalian retina. Exp. Physiol. 2007, 92, 635-640. [CrossRef]

29. Abcouwer, S.F.; Gardner, T.W. Diabetic retinopathy: Loss of neuroretinal adaptation to the diabetic metabolic environment. Ann. N. Y. Acad. Sci. 2014, 1311, 174. [CrossRef]

30. Sohn, E.H.; van Dijk, H.W.; Jiao, C.; Kok, P.H.B.; Jeong, W.; Demirkaya, N.; Garmager, A.; Wit, F.; Kucukevcilioglu, M.; van Velthoven, M.E.J.; et al. Retinal neurodegeneration may precede microvascular changes characteristic of diabetic retinopathy in diabetes mellitus. Proc. Natl. Acad. Sci. USA 2016, 113, E2655-E2664. [CrossRef]

31. Wilkinson-Berka, J.L. Angiotensin and diabetic retinopathy. Int. J. Biochem. Cell Biol. 2006, 38, 752-765. [CrossRef] [PubMed]

32. Silva, K.C.; Rosales, M.A.; Biswas, S.K.; Lopes de Faria, J.B.; Lopes de Faria, J.M. Diabetic retinal neurodegeneration is associated with mitochondrial oxidative stress and is improved by an angiotensin receptor blocker in a model combining hypertension and diabetes. Diabetes 2009, 58, 1382-1390. [CrossRef] [PubMed]

33. Samara, W.A.; Shahlaee, A.; Adam, M.K.; Khan, M.A.; Chiang, A.; Maguire, J.I.; Hsu, J.; Ho, A.C. Quantification of diabetic macular ischemia using optical coherence tomography angiography and its relationship with visual acuity. Ophthalmology 2017, 124, 235-244. [CrossRef] [PubMed]

34. Yi, J.; Liu, W.; Chen, S.; Backman, V.; Sheibani, N.; Sorenson, C.M.; Fawzi, A.A.; Linsenmeier, R.A.; Zhang, H.F. Visible light optical coherence tomography measures retinal oxygen metabolic response to systemic oxygenation. Light Sci. Appl. 2015, 4, e334. [CrossRef]

35. Trick, G.L.; Burde, R.M.; Cordon, M.O.; Santiago, J.V.; Kilo, C. The relationship between hue discrimination and contrast sensitivity deficits in patients with diabetes mellitus. Ophthalmology 1988, 95, 693-698. [CrossRef] 
36. Kurtenbach, A.; Wagner, U.; Neu, A.; Schiefer, U.; Ranke, M.B.; Zrenner, E. Brightness matching and colour discrimination in young diabetics without retinopathy. Vis. Res. 1994, 34, 115-122. [CrossRef]

37. Antonetti, D.A.; Barber, A.J.; Bronson, S.K.; Freeman, W.M.; Gardner, T.W.; Jefferson, L.S.; Kester, M.; Kimball, S.R.; Krady, J.K.; LaNoue, K.F.; et al. Diabetic retinopathy: Seeing beyond glucose-induced microvascular disease. Diabetes 2006, 55, 2401-2411. [CrossRef]

38. Vujosevic, S.; Muraca, A.; Gatti, V.; Masoero, L.; Brambilla, M.; Cannillo, B.; Villani, E.; Nucci, P.; De Cillà, S. Peripapillary microvascular and neural changes in diabetes mellitus: An OCT-angiography study. Investig. Ophthalmol. Vis. Sci. 2018, 59, 5074-5081. [CrossRef]

39. Mase, T.; Ishibazawa, A.; Nagaoka, T.; Yokota, H.; Yoshida, A. Radial peripapillary capillary network visualized using wide-field montage optical coherence tomography angiography. Investig. Ophthalmol. Vis. Sci. 2016, 57, 504-510. [CrossRef]

40. Lim, H.B.; Lee, M.W.; Park, J.H.; Kim, K.; Jo, Y.J.; Kim, J.Y. Changes in ganglion cell-inner plexiform layer thickness and retinal microvasculature in hypertension: An optical coherence tomography angiography study. Am. J. Ophthalmol. 2019, 199, 167-176. [CrossRef]

41. Leung, C.K.; Chan, W.M.; Yung, W.H.; Ng, A.C.K.; Woo, J.; Tsang, M.K.; Tse, R.K.K. Comparison of macular and peripapillary measurements for the detection of glaucoma: An optical coherence tomography study. Ophthalmology 2005, 112, 391-400. [CrossRef] [PubMed]

42. Henkind, P. Symposium on glaucoma: Joint meeting with the national society for the prevention of blindness: New observations on the radial peripapillary capillaries. Investig. Ophthalmol. Vis. Sci. 1967, 6, 103-108.

43. Spaide, R.F.; Fujimoto, J.G.; Waheed, N.K. Optical coherence tomography angiography. Prog. Retin. Eye Res. 2018, 64, 1-55. [CrossRef] [PubMed]

44. Spaide, R.F.; Klancnik, J.M.; Cooney, M.J. Retinal vascular layers imaged by fluorescein angiography and optical coherence tomography angiography. JAMA Ophthalmol. 2015, 133, 45-50. [CrossRef]

45. Kergoat, H.; Hérard, M.E.; Lemay, M. RGC sensitivity to mild systemic hypoxia. Investig. Ophthalmol. Vis. Sci. 2006, 47, 5423-5427. [CrossRef]

46. Spaide, R.F.; Fujimoto, J.G.; Waheed, N.K. Image artifacts in optical coherence angiography. Retina 2015, 35, 2163. [CrossRef] 